\title{
Central serous chorioretinopathy
}

\section{J M Jumper}

\section{What is the eye telling us?}

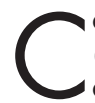
entral serous chorioretinopathy (CSC) has captured the attention of physicians for nearly 150 years. Psychic stress and behavioural traits were originally described as potential contributing factors in the development of this disorder by Horniker in 1927. Since that time, many others have attempted to better define this relation. Gelber and Schatz reported results of detailed psychiatric interviews (which included the gold standard structured interview) with CSC patients. ${ }^{2}$ Of the 33 patients studied, $91 \%$ described a disturbing psychological event immediately preceding their loss of vision. These patients had type A personality scores comparable to patients with myocardial infarction. In 1987, Yannuzzi reported a higher proportion of type A behaviour pattern as measured by the Jenkins Activity Survey in patients with CSC compared to control subjects with other forms of central vision loss. ${ }^{3}$ These two studies would suggest that the constitution of the patient in combination with psychic stress have a role in the development of CSC.

Just as fundus biomicroscopy, angiography, and optical coherence tomography have aided our understanding of the pathological response of the retina and choroid in CSC, our understanding of the psychosocial aspects of disease have been enhanced by standardised questionnaires which have been validated and can be compared to large sample populations of normal subjects. In this issue of the BJO ( $\mathrm{p} \mathrm{704),} \mathrm{Spahn} \mathrm{and}$ coworkers performed psychometric studies (Symptom Checklist, Symptom List, Sixteen Personality Factor Questionnaire, and Questionnaire on Social Support) on patients diagnosed with CSC up to 6 weeks earlier. Thirty seven per cent of these patients had elevated psychic stress within the week before testing. CSC patients were found to have greater social support and no evidence of abnormal psychosomatic symptoms compared to normal sample populations. Personality testing revealed increased emotional instability and insecurity as well as greater spontaneity and flexibility, suggesting to the authors that inner turmoil may be a cause of the stress reaction and an avenue for further investigation. Based on the inconsistent results of all psychological testing performed to date, the authors see no scientific evidence that psychosocial factors have a dominant role in the pathogenesis of CSC.

While this or other studies have not proved the psychogenic induced hypothesis of CSC, an intriguing pattern remains. Repeated studies have shown an association with steroids, both endogenous and exogenous. ${ }^{45}$ Also, CSC has been associated with hypertension and disease states that result in elevated levels of serum cortisol (Cushing's disease) or are commonly treated with steroid medications. ${ }^{467}$ Jampol and coworkers have recently summarised the possible genomic and non-genomic pathways that corticosteroids may affect in the chorioretinal complex in CSC. ${ }^{8}$

CSC remains a unique ophthalmic condition in which a definite link between psychological profile and end organ alterations may one day be made

As previously mentioned, an association between the type $\mathrm{A}$ behaviour pattern (TABP) and CSC has been described..$^{2}$ The TABP has been extensively studied as it relates to cardiovascular disease. Investigators feel that this behaviour pattern acts on the adrenomedullary sympathetic system to alter cardiovascular tone, blood rheology, lipid profiles, and serum hormone levels.' Interestingly, subjects who demonstrate the TABP share many physiological changes that have been described in CSC, including elevated blood pressure and increased serum stress hormones such as cortisol and catecholamines. ${ }^{90}$ Promising research has demonstrated that the TABP is modifiable and that behavioural changes can, in turn, lower the patients' risk of recurrent myocardial infarction. ${ }^{11}$

Is the TABP measured by the psychometric tests performed by Spahn and coworkers? While a controversial issue, the TABP as measured by the Jenkins Activity Survey appears unrelated to factors assessed by the tests reported in this study. In fact, others have shown an inverse correlation between TABP and psychopathology. ${ }^{12}$

CSC remains a unique ophthalmic condition in which a definite link between psychological profile and end organ alterations may one day be made. Our continued attention to this disease is vital. The clinical and psychological findings as well as the neuroendocrine associations may lead to a better understanding not only of CSC but also of other macular and, possibly, vascular diseases. Alternatively, treatments directed at behaviour modification or neuroendocrine alteration in patients with TABP and cardiovascular disease may translate to improved treatments for CSC.

Br J Ophthalmol 2003;87:663

\section{Author's affiliations}

J M Jumper, One Daniel Burnham Court, Suite 210C, San Francisco, CA 94109, USA mikejumper@yahoo.com

\section{REFERENCES}

1 Horniker E. Su di unaforma di retinite centrale di origine vasoneurotica. Ann Ottalmol 1927;55:830-40.

2 Gelber GS, Schatz H. Loss of vision due to central serous chorioretinopathy following psychological stress. Am J Psychiatr 1987; 144:46-50.

3 Yannuzzi LA. Type-A behavior and central serous chorioretinopathy. Retina 1987;7:111-31.

4 Carvalho-Recchia CA, Yannuzzi LA, Negrao $\mathrm{S}$, et al. Corticosteroids and central serous chorioretinopathy. Ophthalmology 2002:109: 1834-7.

5 Garg SP, Dada T, Talwar D, et al Endogenous cortisol profile in patients with central serous chorioretinopathy. $\mathrm{Br} J$ Ophthalmol 1997:81:962-4.

6 Bouzas EA, Scott MH, Mastorakos G, et al. Central serous chorioretinopathy in endogenous hypercortisolism. Arch Ophthalmol 1993;111:1229-33.

7 Tittl MK, Spaide RF, Wong D, et al. Systemic findings associated with central serous chorioretinopathy. Am J Ophthalmol 1999; 128:63-8.

8 Jampol LM, Weinreb R, Yannuzzi L. Involvement of corticosteroids and catecholamines in the pathogenesis of central serous chorioretinopathy: a rationale for new treatment strategies. Ophthalmology 2002; 109:1765-6.

9 Fredrikson M, Blumenthal JA. Serum lipids, neuroendocrine and cardiovascular responses to stress in healthy type A men. Biol Psychol 1992;34:45-58

10 Manuck SB, Garland FN. Coronary-prone behavior pattern, task incentive, and cardiovascular response. Psychophysiology 1979;16:136-42.

11 Friedman $M$, Thoresen CE, Gill JJ, et al. Alteration of type $A$ behavior and its effect on cardiac recurrences in post myocardial infarction patients: summary results of the recurrent coronary prevention project. Am Heart J 1986; 11 12:653-65.

12 Wadden TA, Anderton CH, Foster GD, et al The Jenkins activity survey: does it measure psychopathology? J Psychosom Res 1983;27:321-5. 


\section{Second generation vital stains in retinal surgery}

\section{R B Bhisitkul}

\section{Further innovations may enable novel surgical strategies}

S an article in this issue of the $B J O$ by Stalmans and colleagues ( $p$ 713) and

another recently by Li and colleagues, the novel vital stains trypan blue (TB) and infracyanine green are investigated clinically. These vital stains are promising in vitreoretinal surgery as alternatives to the by now familiar agent indocyanine green (ICG). Firstly, a brief history of membrane staining. ICG, a fluorescent dye used in choroidal angiography, was recognised for its potential in the operating room by cataract surgeons, who first used it to stain the anterior capsule to facilitate capsulorrhexis in difficult cases. Vitreoretinal surgeons, taking notice of their anterior segment colleagues' success, soon found that ICG similarly stained the internal limiting membrane (ILM) of the retina. This was important because peeling of the nearly invisible ILM from the retinal surface had recently been advocated in macular hole surgery ${ }^{2}$; this was easier said than done, however, until the arrival of ICG made ILM peeling easier, faster, and less traumatic. Thus, a trend in posterior segment surgery was born, and ICG soon enjoyed widespread attention. A flurry of articles appeared, with several early clinical series reporting impressive success rates in macular hole surgery with ICG assisted ILM peeling. ${ }^{45}$ ICG staining has by now entered the mainstream of retinal surgery as a useful tool in macular hole repair and epiretinal membrane removal.

But, more recently, some doubts have arisen around ICG, as a critical reassessment has followed the initial enthusiasm. As discussed by the authors of both of these papers, greater clinical experience has begun to reveal possible toxic effects of ICG on the RPE and neural retina. Engelbrecht et al published a clinical series that found central RPE atrophy with poor visual outcomes after some cases of ICG assisted macular hole repair, possibly caused by direct contact of ICG with RPE cells in the area of the hole. ${ }^{6}$ Laboratory studies, in a perhaps inverted sequence, have followed the initial clinical reports and have raised further concerns of overlooked ICG toxicity. Histological and electron microscopic examinations of surgically excised
ILM specimens have demonstrated disruption of retinal elements after ICG application. Muller cell fragments, as well as astrocytes and cellular debris, were observed in ILM specimens peeled with ICG; this was in contrast with those peeled using no $\mathrm{ICG}^{5}{ }^{5}$ suggesting an alteration of the "cleavage plane" between the ILM and underlying neural retina. In vitro studies have indicated toxicity of ICG and its aqueous solvent to cultured RPE cells, ${ }^{89}$ a possible correlate to the clinical study of central RPE atrophy after macular hole surgery using ICG in some patients. ${ }^{6}$ Such concerns have led some surgeons to attempt to minimise ICG exposure during surgery, with reduced concentrations and application times of the dye, as well as circumscribed indications for adjunctive ICG use.

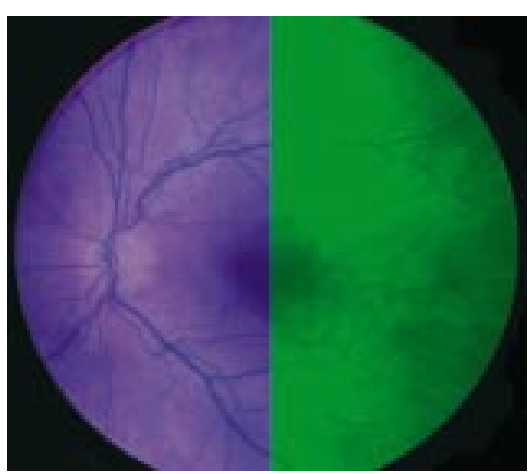

Here's where the work presented by Stalmans et al comes in. They performed double staining with trypan blue and infracyanine green to enhance visualisation during macular pucker surgery. One of two important aspects of this paper is the introduction of infracyanine green as an alternative to indocyanine green for vital staining in posterior segment surgery. Under the theory that retinal toxicity may be a consequence of the hypotonicity of standard indocyanine green in its aqueous solvent, the authors have turned to infracyanine green as an iso-osmotic alternative.

Indocyanine green is prepared for surgical use by reconstituting it with pure water as a solvent, before bringing it to its final concentration in balanced saline solution; it is the hypo-osmolarity of the solvent and the final solution that is suspected by the authors of disrupting cellular elements of the neural retinal. In contrast, infracyanine green, which uses $5 \%$ glucose solution as its solvent for an iso-osmotic final solution, offers comparable staining of the ILM while reducing the untoward osmotic effects. In previous in vitro studies by this group, infracyanine green in 5\% glucose did not demonstrate cytotoxicity to cultured RPE cells, while ICG exposure led to significantly increased cell death. ${ }^{9}$ Similarly, in the present study ILM specimens excised with infracyanine green were not noted to include Muller cell footplates or other evidence of neural retinal disruption when examined by histopathology and electron microscopy. Comparing ICG and infracyanine green, maybe the grass is actually greener on the other side of the fence.

Another central aspect of the paper by Stalmans et al and also that of Li et al is the use of trypan blue for epiretinal membrane surgery. The introduction of trypan blue is more than just a change in colour schemes; its properties as a vital stain are quite distinct from those we're accustomed to with indocyanine green (or even infracyanine green). Unlike the green dyes which selectively stain the acellular ILM but not overlying membranes and vitreous, trypan blue directly stains epiretinal membranes (ERMs), making it valuable in cases such as macular pucker removal. Whereas ICG can only indicate the presence of the ERM by its lack of green staining within an area of stained ILM (referred to as "negative staining"), trypan blue has an affinity for the cellular material composing epiretinal membranes (ERMs), providing visualisation and localisation. One advantage of this is noted by the authors, who found in some cases that trypan blue reveals the extent of the ERM to be much larger than suspected clinically. It is possible that macular pucker surgery unaided by trypan blue can underestimate the size of the membrane, resulting in incomplete ERM removal and residual macular distortion. Trypan blue has other potential surgical benefits; Li et al found that it not only stains ERMs but also provides adequate staining of the ILM, and they forwarded the use of trypan blue as yet another alternative to ICG in macular hole repair with ILM peeling. Finally, in another twist, Stalmans et al exploit the "complementarity" of these dyes in a double staining technique for macular pucker removal, first with trypan blue to peel the ERM, then with infracyanine green to peel the underlying ILM. It would appear that no membrane is safe from vitreoretinal surgeons these days.

The introduction of trypan blue and infracyanine green by no means lays to 
rest the issue of retinal toxicity. Trypan blue stained ILM fragments were found by Li et al to contain glial elements from the neurosensory retina, raising again the spectre of retinal disruption which can affect visual outcomes. As with ICG, with more study and clinical experience the limitations of trypan blue and infracyanine green will be defined while their dosages and application times are optimised. Other barriers also remain to be overcome, not the least of which is that trypan blue and infracyanine green are unavailable for surgical use in many countries including the United States. But the work presented in these two papers marks an important development in this relatively new technology, moving beyond ICG as the sole dye to a second generation of vital stains for intraocular surgery. As more types of vital stains with distinct properties are added to our arsenal, further innovations may enable novel surgical strategies. One can envision in the future an array of vital stains, a sort of surgical palette, with different intraoperative dyes or even non-invasive dyes having distinct affinities for specific membranes or cell types. Diabetic fibrovascular membranes, vitreous cortical hyaloid, proliferative vitreoretinopathy membranes, neurosensory retina, choroidal neovascular membranes, active tumour cells-all might be promising targets for vital stains. Such advances will probably require that we move beyond "off the shelf" agents and instead turn our attention to the research and development of new vital stains specifically for ophthalmic surgery.

Br J Ophthalmol 2003;87:664-665

\section{Author's affiliations}

R B Bhisitkul, UCSF Department of

Ophthalmology 10 Kirkham Street, K301 San

Francisco, CA 94143, USA;

bhisi@itsa.ucsf.edu

\section{REFERENCES}

1 Li K, Wong D, Hiscott $P$, et al. Trypan blue staining of internal limiting membrane and epiretinal membrane during vitrectomy: visual results and histopathological findings. $\mathrm{Br} J$ Ophthalmol 2003;87:216-19.
2 Park DW, Sipperly JO, Sneed SR, et al. Macular hole surgery with internal-limiting membrane peeling and intravitreous air. Ophthalmology 1999;106:1392-8.

3 Brooks HL, Jr. Macular hole surgery with and without internal limiting membrane peeling. Ophthalmology 2000;107: 1939-48.

4 Da Mata AP, Burk SE, Riemann CD, et al. Indocyanine green-assisted peeling of the retinal internal limiting membrane during vitrectomy for macular hole repair. Ophthalmology 2001;108: 1187-92.

5 Kwok AKH, Li WWY, Pang CP, et al. Indocyanine green staining and removal of the internal limiting membrane in macular hole surgery: histology and outcome. Am J Ophthalmol 2001;132:178-83.

6 Engelbrecht NE, Freeman J, Sternberg Jr P, et al. Retinal pigment epithelial changes after macular hole surgery with indocyanine green-assisted internal limiting membrane peeling. Am J Ophthalmol 2002;133: 89-94.

7 Gandorfer A, Haritoglou C, Gass CA, et al. Indocyanine green-assisted peeling of the internal limiting membrane may cause retinal damage. Am J Ophthalmol 2001;132:431-3

8 Sippy BD, Engelbrecht NE, Hubbard GB, et al. Indocyanine green effect on cultured human retinal pigment epithelial cells: implications for macular hole surgery. Am J Ophthalmol 2001;132: 433-5.

9 Stalmans P, Van Aken E, Veckeneer M, et al Toxic effects of indocyanine green on retinal pigment epithelium related to osmotic effects of the solvent. Am J Ophthalmol 2002;134: 282-5.

\section{Too dry or not too dry}

\section{J P Whitcher}

\section{Is that really the question or do eye symptoms lie?}

F or those of us who see patients on a regular basis, how many times a day do we hear the recurring complaint, "Doctor, my eyes feel so dry ... they itch, they burn, they feel constantly irritated"? As doctors we must ask, with a sense of wonder, do all of these patients really have dry eyes? Are we currently in the midst of a global epidemic of ocular dryness that has caught us unawares and unprepared? Our patients are telling us something when they describe their symptoms, but in the process are they also unintentionally misleading us? Are their eyes truly dry, and if they are not, is it possible for us to tell the difference?

Lee et al in the December issue of the $B J O$ have done a superb job of documenting the prevalence of dry eye symptoms in a village population in Sumatra, Indonesia. ${ }^{1}$ Their statistical approach is impeccable. Using a one stage cluster sampling procedure, they randomly selected 100 households in each of five rural villages and one provincial town in Riau Province over a 3 month period in 2001. Demographic, life style, and medical data were collected from 1058 participants and dry eye symptoms were assessed using a six item questionnaire. The questions were, essentially, do your eyes ever feel dry, do you experience grittiness, burning, or redness, do you have crusting, and are your eyes ever stuck shut? The frequency of these symptoms was graded for each individual as rarely, sometimes, often, or all the time. One or more of these symptoms occurring often or all the time was felt to be significant, and those subjects who responded positively to these questions were placed in the dry eye symptom group and included in the analysis. The presence or absence of pterygium in each individual was also documented. Lee et al then submitted their data to detailed statistical analysis and arrived at the conclusion that $27.5 \%$ of all the individuals questioned experienced one or more of the six ocular symptoms often or all the time, and that the only factors found to be associated with these symptoms were a history of current smoking and the presence of a pterygium. Interestingly, the prevalence for dry eye symptoms was 1.4 times greater in men than in women.
Problems arise, however, when dry eye symptoms become by inference dry eye syndromes. As stated in their abstract the aim of the study was "To determine the prevalence and identify associated risk factors for dry eye syndrome in a population in Sumatra, Indonesia." Dry eye syndromes by definition encompass a constellation of diverse disease processes that produce objective signs of keratoconjunctivitis sicca (KCS) with or without a concomitant decrease in tear production. ${ }^{2}$ The classic prototype of the dry eye syndrome is Sjögren's syndrome, but there are many other causes of KCS including cicatrising conjunctival diseases such as trachoma and pemphigoid, non-cicatrising syndromes causing specific dry eye findings, and atypical syndromes such as keratomalacia in which the eye is symptomatically and objectively dry but tear production is paradoxically normal. ${ }^{2}$ The diagnosis of dry eye syndrome or KCS implies that the patient has a specific disease entity as an underlying cause and that the condition is potentially treatable.

\section{The dry eye in most cases is truly a diagnostic dilemma until objective tests are performed}

Lee et al have documented the prevalence of dry eye symptoms in this village population in Indonesia, but they have not provided us with objective proof that any of the subjects in the study actually had signs of KCS. The authors remarked on this shortcoming in their discussion and stated that local sociocultural sensitivities precluded an interventional study 
that would allow objective dry eye tests. This is unfortunate because we still do not know the true prevalence of KCS in this interesting group of patients. With minimal intervention this question could have been answered by performing Schirmer's tests without anaesthesia, fluorescein staining, tear break up time, and rose bengal staining on all the subjects. Using these objective tests the true prevalence of dry eyes in this population could have been easily determined.

Why is it necessary to perform objective tests? Don't the patients' symptoms give us a good indication of whether or not they really have dry eyes? Lee et al have provided the answer in Table 3 of their article. The relation between symptoms of dryness and objective findings of dryness is like comparing apples and oranges. Ask the same individuals whether or not their eyes feel dry and then perform objective tests, and you find as much as a sevenfold difference in the outcome (14.6\% with symptoms, $2.0 \%$ with rose bengal staining). ${ }^{3}$ In some populations that were questioned about their dry eye symptoms, the prevalence rate was as high as $28.7 \%$ (Table 3). ${ }^{4}$ How can there be such a disparity between the symptoms of dryness and the objective findings of dryness? The simple answer is that the eye is not very smart. When it is stressed it has a very limited range of symptomatic responses-pain, foreign body sensation, burning, itching, dryness. Any of these symptoms can be caused by any number of ocular conditions from infections to allergic reactions to something as simple as conjunctival concretions. It is important to always invoke the innocent until proved guilty principle when suspecting a dry eye. Until ocular dryness can be proved by objective tests, mere testimony (symptoms) can only be used to raise our suspicion that it may exist.

The dry eye in most cases is truly a diagnostic dilemma until objective tests are performed. For years we asked patients in our dry eye clinic a standard series of questions. Those patients both with and without an objective diagnosis of ocular dryness gave positive answers to questions regarding foreign body sensation, burning, redness, inability to tear, itching, etc. It wasn't until we retrospectively looked at 304 patients with objectively proved KCS and compared them with 195 patients with ocular complaints but who did not have proved KCS that we realised it was the specificity of the questions that was important, not the sensitivity. So what was the most important question to ask a patient who may have a dry eye? "Can you cry?" Patients who were unable to produce tears even under stressful conditions were very likely to have a true dry eye syndrome compared to those who were symptomatic but were not actually dry ( $\mathrm{p}$ value $<0.00001$ ). ${ }^{5}$

Questions about ocular symptoms of dryness are definitely important, but only as corroborative evidence to point the way to objective tests. Is the eye guilty of being dry? It must remain innocent until proved otherwise beyond the shadow of a doubt. That is the dilemma. Is the eye too dry or not too dry? The answer to this question only serves to guide us as doctors to the objective tests with which we can ultimately determine the true diagnosis.

Br J Ophthalmol 2003;87:665-666

Author's affiliations

J P Whitcher, UCSF, Department of Ophthalmology, 95 Kirkham Street, San Francisco, CA 94143-0944, USA; nepal@itsa.ucsf.edu

\section{REFERENCES}

1 Lee AJ, Lee J, Saw S-M, et al. Prevalence and risk factors associated with dry eye symptoms: a population based study in Indonesia. $\mathrm{Br} J$ Ophthalmol 2002;86:1347-51.

2 Whitcher JP. Clinical diagnosis of the dry eye. Int Ophthalmol Clin 1987;27:7-24. 3 Schein OD, Munoz B, Tielsch JM, et al. Prevalence of dry eye among the elderly. Am J Ophthalmol 1997; 124:723-8.

4 Coffrey BE, Richter D, Simpson T, et al. The Canadian dry eye epidemiology study. In: Sullivan DA, et al, eds. Lacrimal gland, tear film, and dry eye syndromes 2. New York: Plenum Press, 1998:805-6

5 Whitcher JP, Gritz DC, Daniels TE. The dry eye: a diagnostic dilemma. Int Ophthalmo Clin 1998;33:23-37. 